\title{
Nox4-dependent ROS modulation by amino endoperoxides to induce apoptosis in cancer cells
}

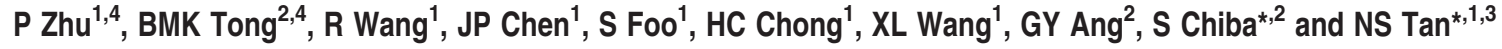

Tumor metastasis is the main cause of death in cancer patients. Anoikis resistance is one critical malefactor of metastatic cancer cells to resist current clinical chemotherapeutic treatments. Although endoperoxide-containing compounds have long been suggested as anticancer drugs, few have been clinically employed due to their instability, complex synthesis procedure or low tumor cell selectivity. Herein, we describe a one-pot strategy to synthesize novel amino endoperoxides and their derivatives with good yields and stabilities. In vitro cell-based assays revealed that 4 out of the 14 amino endoperoxides selectively induce metastatic breast carcinoma cells but not normal breast cells to undergo apoptosis, in a dose-dependent manner. Mechanistic studies showed that the most potent amino endoperoxide, 4-Me, is selective for cancer cells expressing a high level of Nox4. The anticancer effects are further shown to be associated with reduced $\mathrm{O}_{2}^{-}: \mathrm{H}_{2} \mathrm{O}_{2}$ ratio and increased. $\mathrm{OH}$ level in the cancerous cells. Animal study showed that 4-Me impairs orthotopic breast tumor growth as well as tumor cell metastasis to lymph nodes. Altogether, our study suggests that anticancer strategies that focus on redox-based apoptosis induction in tumors are clinically viable.

Cell Death and Disease (2013) 4, e552; doi:10.1038/cddis.2013.68; published online 21 March 2013

Subject Category: Cancer

Cancer metastasis is an extremely complex process, which occurs through a series of sequential, interrelated and selective steps that include the tumor cell invasion of adjacent tissues, intravasation, traveling through the circulatory system, extravasation, and establishment of new foci of malignancy at a secondary site. ${ }^{1,2}$ Despite significant improvements in diagnosis, surgical techniques, general patient care, and local and systemic adjuvant therapies, $90 \%$ of human cancer deaths are due to metastases, which are resistant to conventional therapies. ${ }^{3}$ Apoptosis is a determinant factor modulating metastasis efficiency. As a barrier to metastases, cells normally undergo apoptosis after they lose contact with the extracellular matrix (ECM), a cell death process termed anoikis. Anoikis resistance, the ability to survive in the absence of normal matrix components, represents a crucial property of metastatic cells that permit malignant tumor cells to survive at crucial steps in the metastasis pathway. ${ }^{4}$ Acquisition of anoikis resistance is a prerequisite for the initial step of metastatic dissemination, which requires the detachment of epithelial tumor cells from the ECM, as well as for cancer cell survival while traversing through the lymphatic and circulatory systems into the secondary tumor sites. Although the signaling mechanisms involved in anoikis resistance are still not completely understood, it has long been implicated as a potent target for cancer treatment due to its crucial importance for metastasis.

Tumors exhibit excessive and persistent elevation of reactive oxygen species (ROS, specifically the superoxide anion $\mathrm{O}_{2}^{-}$), and utilize a redox-based mechanism to evade death by anoikis. ${ }^{5,6}$ Furthermore, a deregulated ROS production leads to cell transformation and aggressive tumor metastasis. ${ }^{7,8}$ Recent findings showed that tumor cells maintain a relatively high $\mathrm{O}_{2}^{-}: \mathrm{H}_{2} \mathrm{O}_{2}$ ratio to confer resistance to anoikis. ${ }^{9}$ Conceivably, therapies that reduce this ratio would sensitize tumor cells to apoptosis by anoikis and severely attenuate metastasis. Although $\mathrm{O}_{2}^{-}$and $\mathrm{H}_{2} \mathrm{O}_{2}$ elicit moderate activities with other biological molecules, the end product of their reaction generates hydroxyl radical $(\cdot \mathrm{OH})$, which is highly reactive, and accounts for most of the oxidative damages attributed to ROS. ${ }^{10}$ Much effort has been put into the discovery of novel antitumor drugs, however, few has been specifically exploited through the redox-based apoptosis as an anticancer therapeutic strategy.

\footnotetext{
${ }^{1}$ School of Biological Sciences, Nanyang Technological University, Singapore, Singapore; ${ }^{2}$ Division of Chemistry and Biological Chemistry, School of Physical and Mathematical Sciences, Nanyang Technological University, Singapore, Singapore and ${ }^{3}$ Institute of Molecular and Cell Biology, Proteos, A ${ }^{\star}$ STAR, Singapore

*Corresponding author: S Chiba, Division of Chemistry and Biological Chemistry, School of Physical and Mathematical Sciences, Nanyang Technological University, Singapore 637551, Singapore. Tel: +65 6513 8013; Fax: + 656791 1961; E-mail: shunsuke@ @ntu.edu.sg

Or NS Tan, School of Biological Sciences, Nanyang Technological University, 60 Nanyang Drive, Singapore 637551, Singapore. Tel: +65 63162941 ; Fax: + 656791 3856; E-mail: nstan@ntu.edu.sg

${ }^{4}$ These authors contributed equally to this work.

Keywords: Nox4; cancer; anoikis; reactive oxygen species; amino endoperoxides

Abbreviations: $\triangle \mathrm{FAD} / \mathrm{NADPH}, \mathrm{FAD}$ deletion mutant; $\triangle \mathrm{HEME}$, HEME deletion mutant; · OH, hydroxyl radical; BMPO, 5-butyl-5-methyl-1-pyrroline 1-oxide; COX-2, cyclooxygenase-2; DEPMPO, 5-(diethoxyphosphoryl)-5-methyl-1-pyrroline-N-oxide; DMTU, dimethylthiourea; ECM, extracellular matrix; EPR, electron paramagnetic resonance spectroscopy; $\mathrm{H}_{2} \mathrm{O}_{2}$, hydrogen peroxide; Nox4, NADPH oxidase 4; LC-MS/MS, liquid chromatography-tandem mass spectroscopy; MCLA, 2-methyl-6-(4methoxyphenyl)-3, 7-diphdroimidazo [1, 2-a] pyrazin-3-one hydrochloride; $\mathrm{NAC}, \mathrm{N}$-acetyl cysteine; $\mathrm{O}_{2}^{-}$, superoxide; poly-HEMA, poly-2-hydroxyethyl methacrylate; $\mathrm{PI}$, propidium iodide; ROS, reactive oxygen species; SPR, surface plasmon resonance; TA, terephthalic acid

Received 28.8.12; revised 27.12.12; accepted 30.1.13; Edited by A Finazzi-Agró
} 
Naturally-occurring as well as synthesized endoperoxides exhibit antimalaria, antiviral and/or anticancer activities (Supplementary Figure S1). ${ }^{11,12}$ The endoperoxide moiety has an important role via the $\mathrm{O}-\mathrm{O}$ bond homolytic cleavage. ${ }^{13,14}$ For example, the antimalarial drug artemisinin has been reported to cause cancer cell death via the upregulation of ROS, which leads to mitochondrial membrane depolarization, activation of caspase- 3 and -7 , and degradation of DNA. ${ }^{15,16}$ There has been considerable interest to explore synthetic methods of endoperoxides with novel structures and properties, while the stability of the weak/reactive $\mathrm{O}-\mathrm{O}$ bond render its installation and further functionalization difficult and challenging. Although several synthetic approaches toward endoperoxides have been exploited, ${ }^{17,18}$ there remains a need for conceptually novel and straightforward methodologies for chemical synthesis of endoperoxides from readily available building blocks.

Herein, we demonstrated a concise one-pot synthesis of novel amino endoperoxides starting from easily available benzonitriles and aryl Grignard reagents through a copper (Cu)-catalyzed aerobic $\mathrm{C}-\mathrm{H}$ oxygenation reaction. We revealed that four amino endoperoxides display significant anticancer action against human breast carcinoma MDA-MB231, but have little cytotoxic effects on normal breast cells. Affinity pulldown followed by liquid chromatography-tandem mass spectroscopy (LC-MS/MS) identified NADPH oxidase 4 (Nox4) as a target of the most potent amino endoperoxide, 4-Me. We further showed that the anticancer effects are associated with reduced $\mathrm{O}_{2}^{-}: \mathrm{H}_{2} \mathrm{O}_{2}$ ratio and increased $\cdot \mathrm{OH}$ level in cancer cells. The treatment of mice bearing orthotropic human breast tumor with 4-Me attenuated the in vivo growth of primary tumor associated with increased apoptosis. The present study showed that endoperoxides induced redoxbased apoptosis in tumors.

\section{Results}

Design and synthesis of novel amino endoperoxides and their derivatives. Using a one-pot strategy we were able to synthesize 14 novel amino endoperoxides and their derivatives with good yields and stabilities (Figure 1). Four of the 14 novel endoperoxide derivatives were found to possess prominent anticancer potentials, which will be described in the subsequent result sections. The synthetic processes of these endoperoxides and yields are presented in Figure 1a, and the structures of the 14 newly designed endoperoxide derivatives with various side chain modifications in Figure $1 \mathrm{~b}$. The detailed synthetic procedures and spectral data are available in Supplementary Results (pages 1-40 of Supplementary Information). Amino endoperoxides selectively cause cancer cell
apoptosis in vitro. The anticancer potential of these novel
amino endoperoxide derivatives was evaluated in human
breast carcinoma cells, MDA-MB-231, while normal breast
cell line MCF-10A was used as a normal cell control. First, we
measured the effects of the 14 individual compounds on the
adhered MDA-MB-231 cells. Interestingly, $0.5 \mathrm{~h}$ treatment
with 6 out of the 14 compounds, namely (1) $4-\mathrm{Me}$, (2) $\mathrm{H}$,
(3) $4-\mathrm{CF}_{3}$, (8) $3-\mathrm{Cl}$, (12) $2^{\prime}$-OMe, and (14) $\mathrm{OH}$ significantly increased apoptosis of MDA-MB-231 cells in a dosedependent manner (Figure 2a, Supplementary Figure S2A), but little effect was observed with the other eight compounds (data not shown), indicating that not only the endoperoxide bridge, but also the side groups of the compounds have important roles in their anticancer potentials. Among the six cancer cell-sensitive amino endoperoxides, (1) 4-Me, (2) $\mathrm{H}$, (3) $4-\mathrm{CF}_{3}$, and (8) 3-Cl, elicited little toxicity against MCF-10A cells (Figure $2 \mathrm{a}$ and Supplementary Figure S2B), suggesting selectivity of these compounds against breast cancer cells MDA-MB-231. In contrast, both of the amino endoperoxides (12) 2'-OMe and hydroxy endoperoxide (14) $\mathrm{OH}$ displayed high toxicity to normal breast cells MCF-10A, and therefore were excluded as suitable anticancer candidates for further studies (Figure 2a and Supplementary Figure S2B). As anoikis resistance is a hallmark of metastatic cancer, which can be a practical target for antimetastatic drugs, we questioned whether the four active compounds could also exert potential effects to interfere with cancer cells anoikis resistance. Therefore, we subjected the cancer cells into a suspension state in the presence of the four individual amino endoperoxides. Interestingly, the four amino endoperoxides caused significantly higher apoptosis of the cancer cells in a dose-dependent manner when compared with vehicle treatment (Figure $2 \mathrm{~b}$ and Supplementary Figure S2C). Among them, 4-Me $(500 \mu \mathrm{M})$ displayed the most potent anticancer action, causing $\sim 32 \%$ and $\sim 44 \%$ apoptotic cells in attached and suspended MDA-MB-231cells, respectively (Figure 2, Supplementary Figures S2A and S2C). At lower concentration of $1 \mu \mathrm{M}$, the $4-\mathrm{Me}$ induced $\sim 7 \%$ and $\sim 11 \%$ of apoptosis on cancer cells within $0.5 \mathrm{~h}$ of adherent and suspension treatments, respectively. The apoptotic effect of 4-Me was more pronounce with $\sim 19-31 \%$ of cancer cells undergoing apoptosis when exposed for a longer period of $12-24 \mathrm{~h}$ (Supplementary Figures S2D and S2E), suggesting the potential of 4-Me as a pharmacological agent. Furthermore, in consistent with adherent results, little cytotoxicity was observed of the four amino endoperoxides on suspended normal breast cells MCF-10A (Figure $2 b$ and Supplementary Figure S2B), corroborating that the apoptosis-inducing effects of these four amino endoperoxides were selective for breast cancer cells, but not for normal breast cells.

Amino endoperoxides targets Nox4 to cause cancer cell death. To understand the mechanism underlying the apoptotic effects of these amino endoperoxides, we sought to identify potential intracellular targets of amino endoperoxide (4-Me) by affinity pulldown assay with the total protein extract of cancer cell MDA-MB-231 lysate (Figure 3a). We designed and synthesized a 4-Me derivative bearing an alkyne side chain (designated as 4'-alkyne; see pages 9, 10, 39 and 40 of Supplementary information), and also employed CLICK-chemistry to establish the pulldown assay. Interestingly, LC-MS/MS analysis consistently identified Nox4 (Supplementary Figure S3A) as the molecular target of 4-Me, but not MCF-10A cells, which was further confirmed by immunoblot analysis (Figure 3b). Next, we expressed several variants of human Nox4 protein, namely FL (full-length protein), $\triangle \mathrm{FAD} / \mathrm{NADPH}$ (FAD deletion mutant), and $\triangle \mathrm{HEME}$ (HEME deletion mutant), and then purified the recombinant 
a

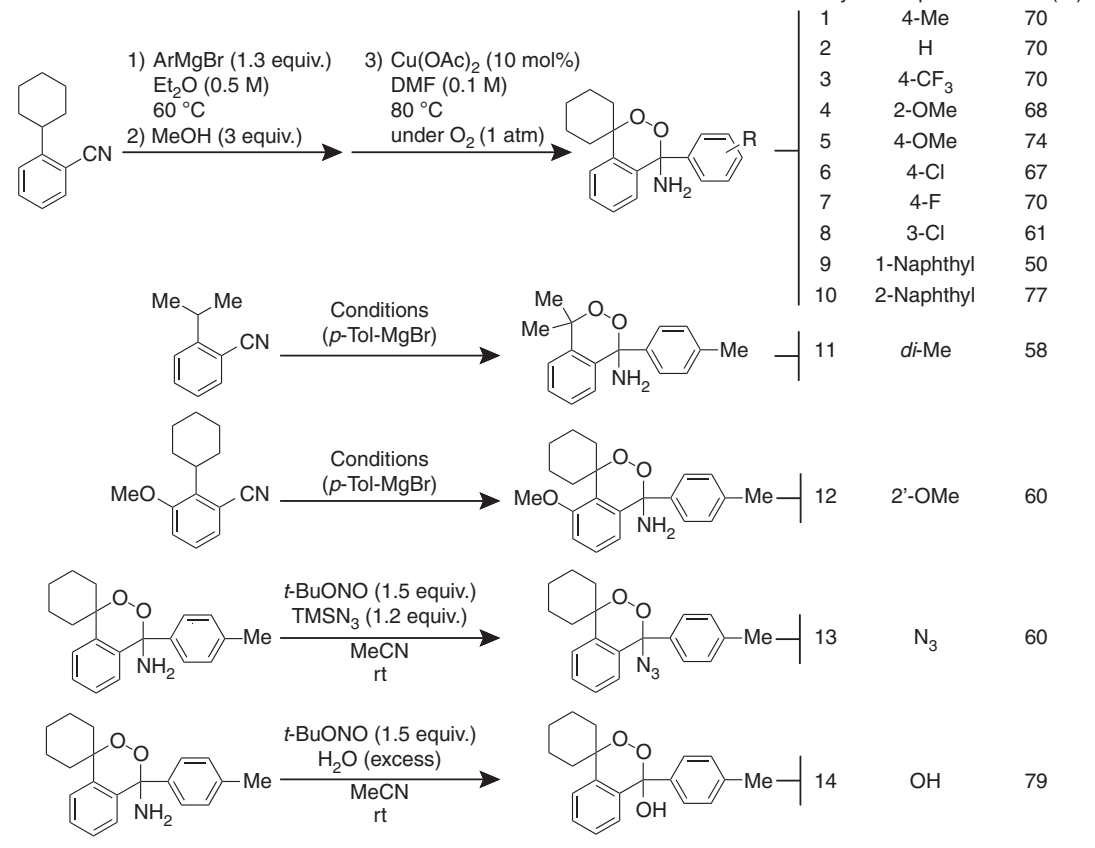

b

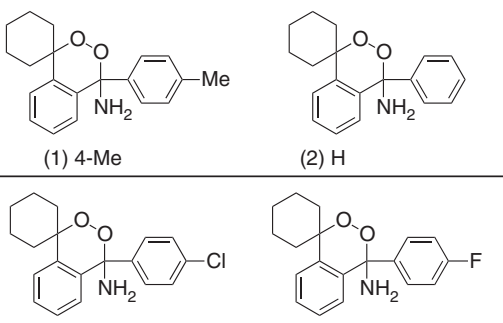

(6) $4-\mathrm{Cl}$

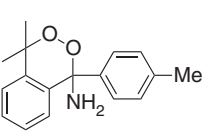

(11) di-Me

(7) $4-\mathrm{F}$
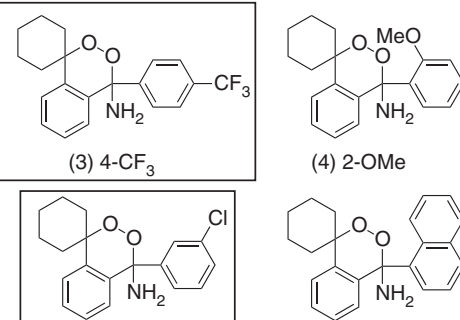

(8) $3-\mathrm{Cl}$

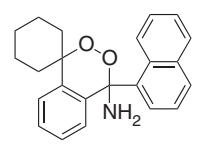

(9) 1-Naphthyl

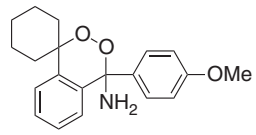

(5) $4-\mathrm{OMe}$

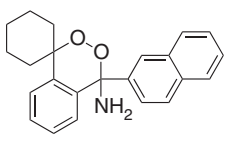

(10) 2-Naphthyl

Figure 1 Synthesis and structures of 14 novel amino endoperoxides and their derivatives. (a) The synthetic conditions and yields of 14 amino endoperoxides and their derivatives. (b) The structures of the 14 novel amino endoperoxides and their derivatives

proteins for surface plasmon resonance (SPR) analyses (Supplementary Figure S3B). Our SPR analyses showed that amino endoperoxides, 4- Me, 4- $\mathrm{CF}_{3}, 3-\mathrm{Cl}$, and $\mathrm{H}$ interacted with full-length Nox4 $\left(\mathrm{K}_{\mathrm{D}}: 4-\mathrm{Me}, 5.67 \times 10^{-7}\right.$; 4-CF3, $7.22 \times 10^{-6} ; 3-\mathrm{Cl}, 5.07 \times 10^{-6} ; \mathrm{H}, 1.05 \times 10^{-6} \mathrm{M}$; Figures $3 \mathrm{c}$ and $\mathrm{d}$ ). Further analysis showed that 4-Me interacted with the HEME domain of Nox4 (Figure 3e). Our findings showed that 4-Me targets Nox4 in breast cancer cell MDA-MB-231, but not in MCF-10A, whose endogenous expression was very low (Figure $3 \mathrm{f}$ ). Next, we performed Nox4 knockdown experiment on MDA-MB-231 cells, generating Nox4-deficient line, MDA-MB-231 Nox4 siRNA. MDAMB-231 transfected with scrambled siRNA served as control (MDA-MB-231 control; Supplementary Figure S3C). Interestingly, the apoptotic effect of 4-Me was significantly diminished in the Nox4-deficient MDA-MB-231 (MDA-MB231 Nox4 siRNA) cells as compared with MDA-MB-231 control cells (Figure $3 g$ and Supplementary Figure S3D), indicating proficient expression of Nox4 is essential for the apoptosis-inducing effect of 4-Me. Although endoperoxides are good substrates for the peroxidase component of cyclooxygenase-2 (COX-2), COX-2 was not identified in our LC-MS/MS analyses. To further confirm this observation, we performed SPR using COX-2 and 4-Me. The result showed that the 4-Me did not interact with COX-2 (Supplementary Figure S3E). Conceivably, cancer cell lines expressing elevated level of Nox4 would be more susceptible to the anticancer action of these endoperoxides when compared with low Nox4-expressing cell lines. Thus, we examined the mRNA and protein expression levels of Nox4 

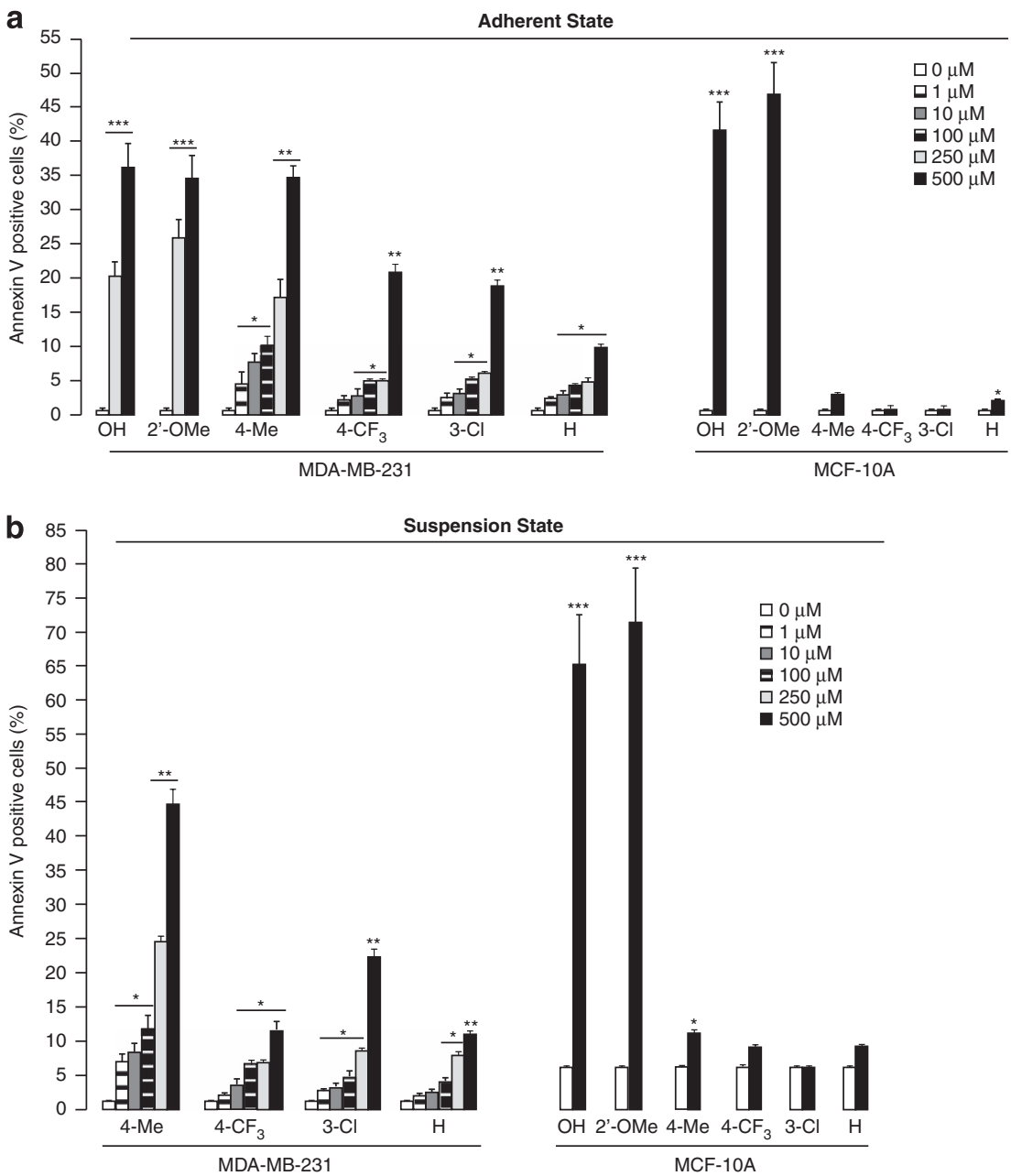

Figure 2 In vitro anticancer effects of amino endoperoxides and their derivatives. (a, b) Percentage of Annexin V positive (apoptotic) cells in adhered (a) and suspended (b) MDA-MB-231 and MCF-10A cells treated with indicated amino endoperoxide compounds and their derivatives under indicated concentrations for $0.5 \mathrm{~h}$. See Supplementary Figure S2 for detailed FACS. Experiments were independently performed three times with at least three replicates of each sample. The concentration 0 indicates vehicle control. Error bars: S.E.M. Comparison was performed against cognate vehicle controls. ${ }^{*} P<0.05$, ${ }^{* *} P<0.01 ;{ }^{* \star *} P<0.001$

in several cell lines. Real-time $\mathrm{GPCR}$ and immunoblot analyses revealed that non-tumorigenic human keratinocyte $\mathrm{HaCaT}$ and metastatic squamous cell carcinoma A-5RT3 express very low (negligible) levels of Nox4 mRNA (Figure 3f). Concordantly, 4-Me did not induce apoptosis in A-5RT3 (Figure $3 \mathrm{~g}$ and Supplementary Figure S4A). Consistent with our above results, the ectopic expression of Nox4 in human embryonic kidney 293 cells rendered these cells susceptible to the apoptotic effect of $4-\mathrm{Me}$ (Supplementary Figures S4B and S4C). Taken together, our findings suggest that amino endoperoxide 4-Me specifically targets high Nox4-expressing cancer cells.

Amino endoperoxides modulate ROS levels in cancer cells. Nox4-dependent ROS production is important for the survival of many cancer types. ${ }^{19,20}$ Nox4 has been reported to predominantly produce $\mathrm{H}_{2} \mathrm{O}_{2}$ more than $\mathrm{O}_{2}^{-}{ }^{21}$ Thus, it is conceivable that the four amino endoperoxides, 4-Me, $4-\mathrm{CF}_{3}$, $3-\mathrm{Cl}$ and $\mathrm{H}$ mediated their anticancer effects by regulating the ROS levels. To test this hypothesis, we first evaluated whether the four amino endoperoxides also modulate ROS level in cancer cells. Treatment of normal breast cells MCF$10 \mathrm{~A}$ with the four amino endoperoxides showed little effect on the ROS level (Figure 4a). However, $0.5 \mathrm{~h}$ treatment of breast cancer cells MDA-MB-231 significantly increased the ROS levels in a dose-dependent manner (Figure 4a). Thus, we measured the level of $\mathrm{H}_{2} \mathrm{O}_{2}$ in cancer cells in the presence of a specific catalase inhibitor, 3-amino-1, 2, 4,triazole. ${ }^{22,23}$ The $\mathrm{H}_{2} \mathrm{O}_{2}$ levels were greatly increased in all of the four amino endoperoxide-treated MDA-MB-231 cells ( 3.5-fold increase with $500 \mu \mathrm{M}$ of 4-Me; Figure 4b). Next, we measured $\mathrm{O}_{2}^{-}$levels by a chemiluminescence assay using 2-methyl-6-(4-methoxyphenyl)-3, 7-dihydroimidazo[1, 2-a] pyrazin-3-one hydrochloride (MCLA). $\mathrm{O}_{2}^{-}$levels were also elevated in amino endoperoxide-treated cells when compared with vehicle control, albeit with a much smaller fold change ( $\sim$.6-fold increase with $500 \mu \mathrm{M}$ of 4-Me; Figure 4c). Using electron paramagnetic resonance spectroscopy (EPR) in combination with 5-(diethoxyphosphoryl)-5-methyl-1-pyrroline- $N$-oxide (DEPMPO) spin trapping, the effect of $4-\mathrm{Me}$ 
a

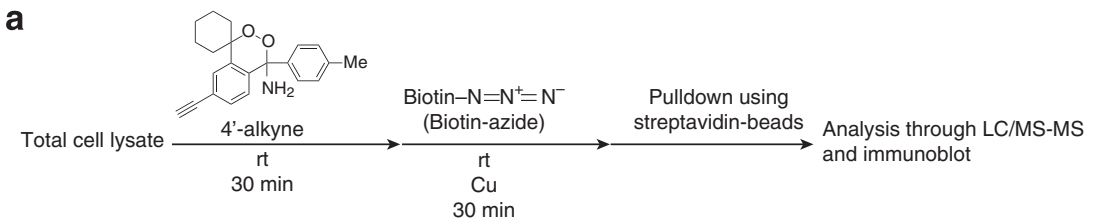

b
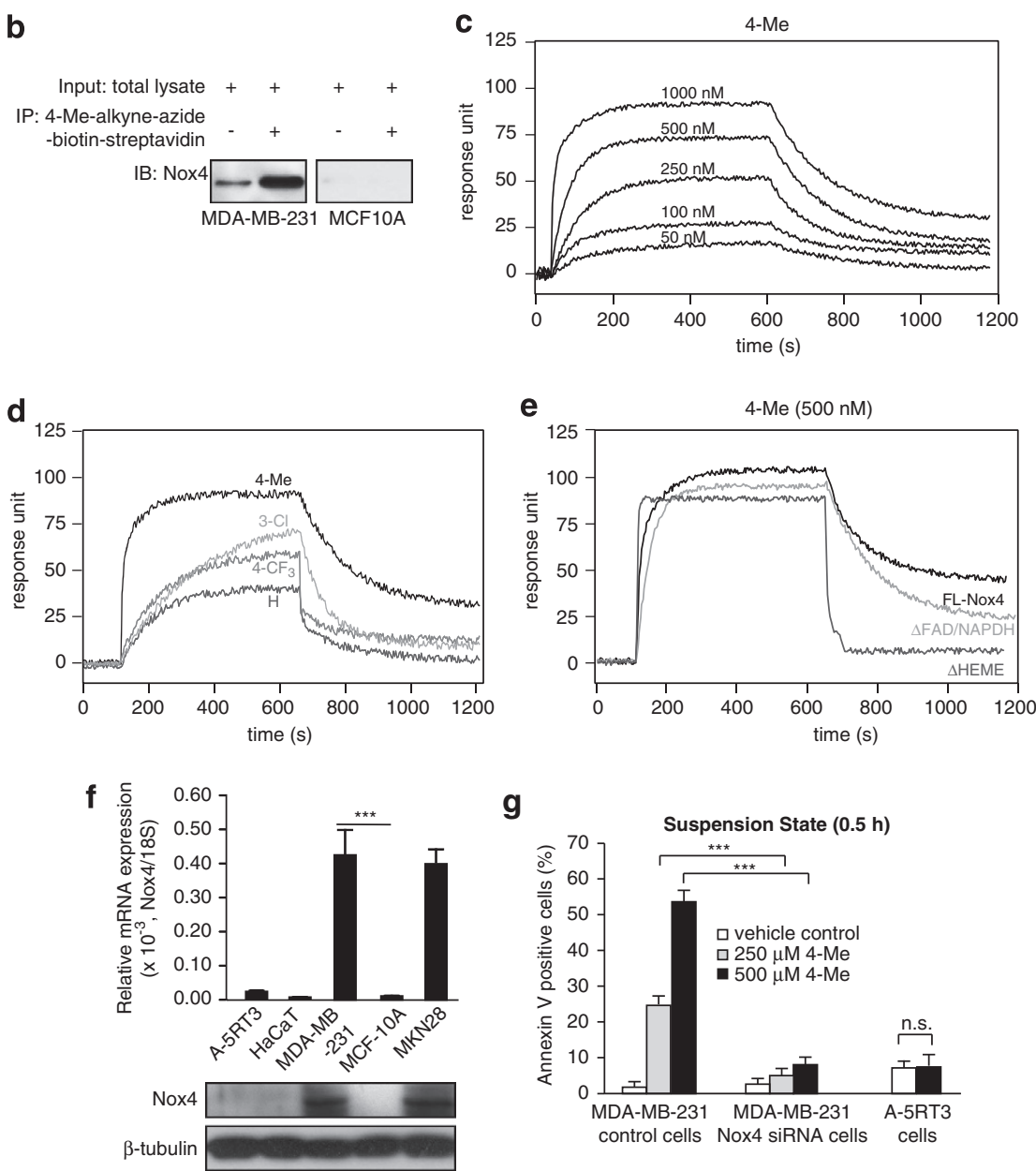

g

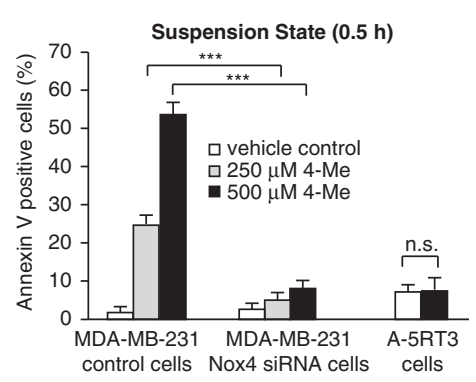

Figure 3 4-Me targets Nox4 to cause apoptosis of MDA-MB-231 cells. (a) Flowchart of the strategy to identify cellular interacting partners of 4-Me. 4-Me with an alkyne group (4'-alkyne) was used as a bait. (b) Immunodetection of Nox 4 from immunoprecipitates of 4'-alkyne conjugated to biotin-azide. 4'-alkyne-azide-biotin was affinity pulldown using streptavidin Sepharose beads. (c-e) Representative sensograms of three independent experiments showing binding profiles between immobilized-full-length (FL) Nox4 and indicated concentrations of 4-Me (c); between immobilized-FL Nox4 and $500 \mathrm{nM}$ of 4-Me, 3-Cl, 4-CF 3 , and $\mathrm{H}$ (d); between either immobilized-FL Nox4, $\Delta \mathrm{FAD} /$ NADPH or $\triangle$ HEME with 4-Me (e). Sensograms were corrected against a reference flow cells with no immobilized proteins. (f) Relative mRNA and protein levels of Nox4 in various human cell lines. $\mathrm{HaCaT}$ is a non-tumorigenic skin line, A-5RT3 is a metastatic skin line, and MKN28 is a gastric carcinoma line. The mRNA data (means \pm S.D.) are from two independent qPCR experiments performed in triplicates. The ribosomal protein $18 \mathrm{~S}$ gene serves as a reference housekeeping gene and $\beta$-tubulin serves as a loading and transfer control in immunoblot. ${ }^{* *} P<0.001$. (g) Percentage of suspension-induced apoptotic (Annexin $V$ positive) cells treated with indicated concentrations of 4-Me for $0.5 \mathrm{~h}$ as analyzed by FACS (5000 events). Vehicle-treated cells served as control. The sum of Annexin $\mathrm{V}^{+} / \mathrm{PI}^{-}$(early apoptosis) and Annexin $\mathrm{V}^{+} / \mathrm{PI}^{+}$ (late apoptosis) cells were considered apoptotic. Results are representative of three independent experiments. See Supplementary Figures S3D and S4A for FACS data. Error bars: S.E.M. Comparison was performed against cognate vehicle controls. n.s. indicates not significant; ${ }^{* \star \star} P<0.001$

was reproduced (Figures $4 \mathrm{~d}$ and e). The $\mathrm{O}_{2}^{-}$level was completely abolished by $\mathrm{O}_{2}^{-}$scavenger Tiron, which served as a negative control for $\mathrm{O}_{2}^{-}$measurement.

The end product of $\mathrm{O}_{2}^{-}$and $\mathrm{H}_{2} \mathrm{O}_{2}$ reaction is $\cdot \mathrm{OH}$, which is responsible for most of the oxidative damage attributed to ROS. $^{10}$ Our measurement using terephthalic acid $(T A)^{24}$ showed a significant increase in $\cdot \mathrm{OH}$ levels ( $\sim$ sixfold with $500 \mu \mathrm{M}$ of 4-Me; Figure 5a). Similarly, the data were reproduced by EPR using 5-tert-butoxycarbonyl-5-methyl-1pyrroline- $\mathrm{N}$-oxide (BMPO) spin trap to detect. $\mathrm{OH}$ adduct (Figures $5 \mathrm{~b}$ and $\mathrm{c}$ ). The $\mathrm{OH}$ quencher dimethylthiourea (DMTU) was used as negative control (Figure 5). Immunoblot analysis also showed that there was significantly higher level of cleaved PARP in 4-Me-treated MDA-MB-231 cells (Supplementary Figure S5A), and no observable difference in cell cycle was detected (Supplementary Figure S5B). 


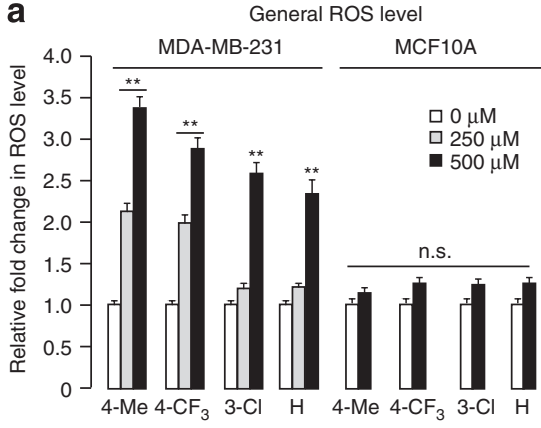

d

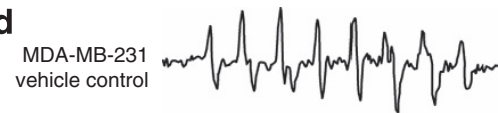

MDA-MB-231
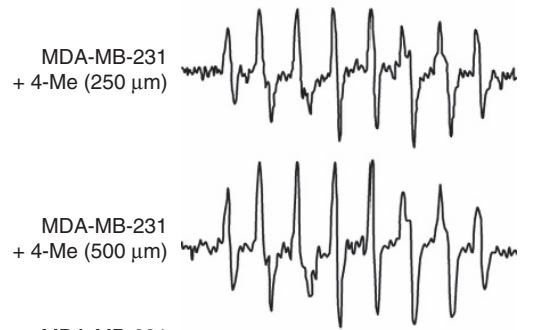

MDA-MB-231

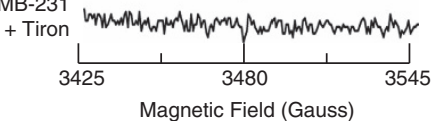

b

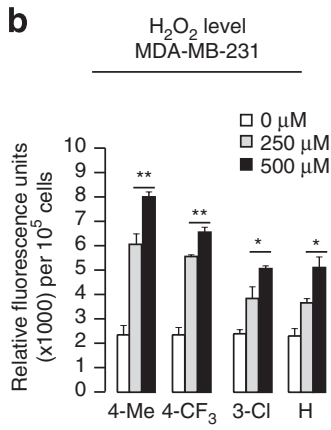

e

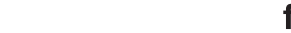

f

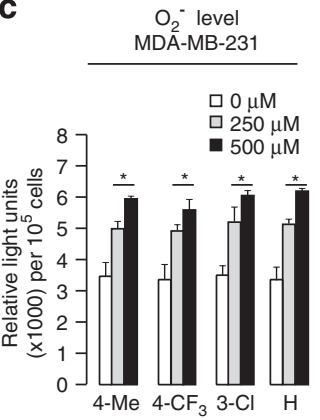

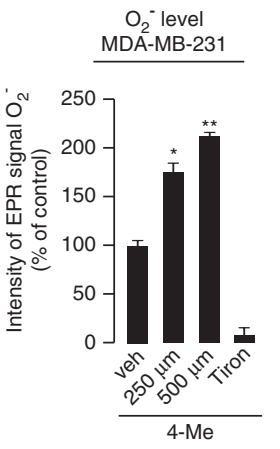

Figure 4 Novel amino endoperoxide compounds regulate ROS levels in cancer cells. (a) General ROS levels (expressed as fold change) in MCF-10A and MDA-MB-231 cells suspended in the presence of the four amino endoperoxide compounds at indicated concentrations for $0.5 \mathrm{~h}$ as measured by CM- $\mathrm{H} 2 \mathrm{DCFDA}$. (b, c) $\mathrm{H}_{2} \mathrm{O}_{2}(\mathbf{b})$ and $\mathrm{O}_{2}^{-}$(c) levels in MDA-MB-231 cells treated like in (a) as determined by Amplex Red and 2-methyl-6-(4-methoxyphenyl)-3, 7-dihydroimidazo[1, 2-a] pyrazin-3-one hydrochloride (MCLA) assays, respectively. (d) Representative EPR spectra of DEPMPO-superoxide spin adduct from MDA-MB-231 cells suspended in the presence of the 4-Me. The superoxide adduct of DEPMPO has hyper fine splitting constants of $a_{N}=13.13 \mathrm{G} ; a_{\mathrm{P}}=55.61 \mathrm{G} ; a^{\beta}{ }_{H}=13.11 \mathrm{G} ; a^{\gamma}{ }_{H}=0.71,0.42,0.7,0.25$, and $0.6 \mathrm{G}$. The EPR spectra represent the averaged signals of 10 scans. (e) EPR signal intensity (expressed as percentage of control) at $3480 \mathrm{G}$ from MDA-MB-231 cells in (d). Tiron-treated measurements serve as negative signal controls. (f) Arbitrary relative $\mathrm{O}_{2}^{-}: \mathrm{H}_{2} \mathrm{O}_{2}$ ratios calculated based on $\mathrm{H}_{2} \mathrm{O}_{2}$ (b) and $\mathrm{O}_{2}^{-}$(c) values are shown in boxes. Each experiment was independently performed three times with triplicates. The concentration 0 indicates vehicle control and its ROS value was set as 1. Error bars: S.E.M. Comparison was performed against cognate vehicle controls. n.s. represents not significant, ${ }^{*} P<0.05,{ }^{* \star} P<0.01$
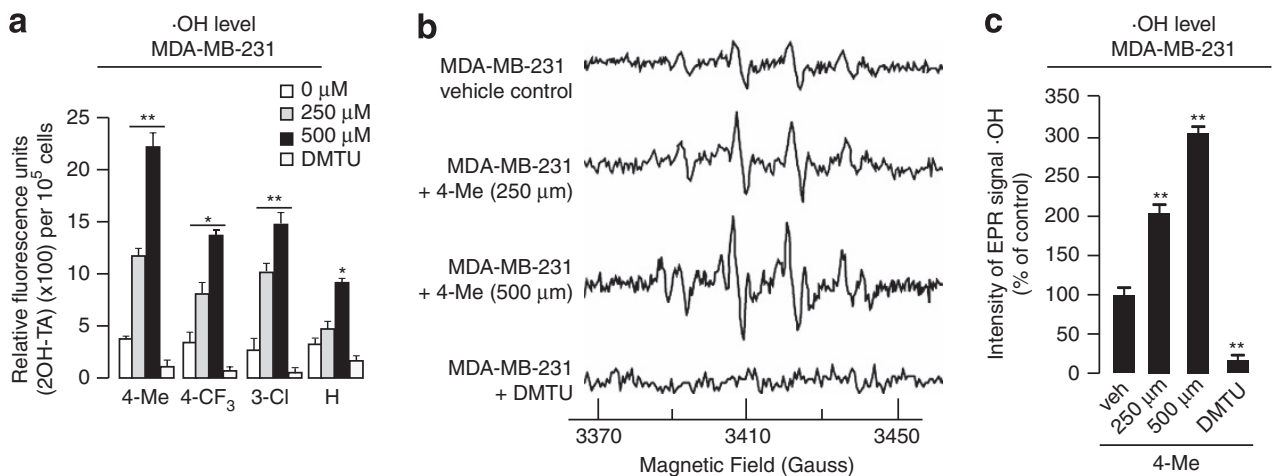

Figure 5 Amino endoperoxide compounds upregulate $\mathrm{OH}$ levels in cancer cells. (a) Levels of $\mathrm{OH}$ (expressed as fold change) in MDA-MB-231 cells suspended in the presence of the four amino endoperoxides at indicated concentrations for $0.5 \mathrm{~h}$ measured using TA. (b) Representative EPR spectra of BMPO-OH spin adduct from MDA-MB231 suspended in the presence 4-Me. For . $\mathrm{OH}$ adduct, spectrometer parameters were: modulation frequency, $100 \mathrm{kHz}$; scan range, $100 \mathrm{G}$; field set, $350 \mathrm{G}$; microwave power, $10 \mathrm{~mW}$; modulation amplitude, $1 \mathrm{G}$; time constant, $5 \mathrm{~ms}$; and scan time $5.14 \mathrm{~s}$. The EPR spectra represent the averaged signals of 10 scans. Dimethylthiourea was used as negative controls for $\cdot \mathrm{OH}$ measurements. (c) EPR signal intensity expressed as percentage of control at $3410 \mathrm{G}$ from MDA-MB-231 cells in (b). Each experiment was independently performed three times with triplicates. The concentration 0 indicates vehicle control and its $\cdot \mathrm{OH}$ value was set as 1. Error bars, S.E.M. Comparison was performed against cognate vehicle controls. n.s. represents not significant, ${ }^{\star} P<0.05,{ }^{* \star} P<0.01$

Our above findings suggest that 4-Me modulates intracellular ROS to elicit its anticancer effects. Concordantly, antioxidant reagent NAC reduced this apoptosis-inducing effect of 4-Me (Supplementary Figures S5C and S5D). The effect of 4-Me on the general ROS, $\mathrm{O}_{2}^{-}: \mathrm{H}_{2} \mathrm{O}_{2}$ ratio and $\cdot \mathrm{OH}$ radical levels were also significantly abolished in the MDA- 
MB-231 Nox4 siRNA cancer cells (Supplementary Figure S6). Taken together, these observations indicate that the amino endoperoxide 4-Me modulated Nox4 activity to alter intracellular ROS, reducing $\mathrm{O}_{2}^{-}: \mathrm{H}_{2} \mathrm{O}_{2}$ ratio and increasing $\cdot \mathrm{OH}$ levels in cancer cells, which lead to the apoptosis of Nox4-proficient cancer cells.

Redox-based anticancer action of amino endoperoxide (4-Me) impairs orthotopic tumor growth. As shown above, the four endoperoxides elicited prominent in vitro anticancer effects through regulation of cellular ROS environment. Next, we set out to investigate their in vivo therapeutic effects on tumor development by using the MDAMB-231 orthotopic nude mice model. Two millions of MDAMB-231 cells were transplanted into one of the fourth mammary fat pad of individual 6-week-old female nude mice. The mice were randomly divided into two groups $(n=5$ each group) 8 days post-transplantation of tumor cells, and the two groups of mice were intraperitoneally treated with either $3.2 \mathrm{mg} / \mathrm{kg}$ of amino endoperoxide (4-Me) or control vehicle once every 3 days. As expected, the injection of MDA-MB-231 cells into immunodeficient mice induced large primary mammary tumors $\left(\sim 800 \mathrm{~mm}^{3}\right)$ in all of the five vehicle control mice at day 36 . Interestingly, the 4-Me treatment exerted significant inhibition of the tumor growth in the other group of mice treated with amino endoperoxide, displaying an $\sim 75 \%$ reduction in tumor size (Figures $6 a$ and $b$ top panel). Notably, amino endoperoxide (4-Me) also significantly reduced lymph node metastasis in nude mice, as compared with vehicle treatment (Figure $6 \mathrm{~b}$ bottom panel). All of the mice were well tolerated without obvious signs of drug-related toxicity throughout the course of this study, even at $16-32 \mathrm{mg} / \mathrm{kg}$ dosages (equivalent to $51.5-103 \mu \mathrm{mol} / \mathrm{kg}$ ) as evidenced by undetectable necrotic tissue damage and expression of active cleaved caspase-3 (Supplementary Figure S7). In addition, immunofluoresence (Figure 6c) and immunoblot (Figure 6d) analyses of the tumor biopsies indicated significantly reduced cell proliferation and enhanced cell apoptosis in 4-Me-treated mice tumors as compared with vehicle-treated mice tumors. Together, these observations clearly support the antimetastatic cancer effect of amino endoperoxides, particularly, 4-Me.

\section{Discussion}

Cancer metastasis remains the primary culprit of patient deaths worldwide, despite all the tremendous efforts put into basic and clinical cancer research. Breast cancer is the leading cause of cancer death among women with $\sim 1.38$ million new cases and an estimated 458000 deaths each year. ${ }^{25}$ One-third of patients who present with earlystage breast cancer will go on to develop metastatic breast cancer. $^{26}$ Metastatic cancer therefore presents a major clinical problem in oncology. Despite the many moleculartargeted drugs, which either have low cancer cell selectivity or benefits only a small subset of cancer patients, early (before metastasis forms) detection remains the primary advantage for cancer treatment. The four amino endoperoxides, 4-Me, 4- $\mathrm{CF}_{3}, 3-\mathrm{Cl}$, and $\mathrm{H}$ have advantageous selectivity against high Nox4-expressing cancer cells, exhibiting prominent clinical potential. Although endoperoxide-containing compounds either from nature or artificially synthesized have been reported to exhibit potent anticancer potentials, they suffer from instability, complicated synthetic protocol, low efficiency in terms of chemical synthetic yields, and poor tissue selectivity, thus making them unattractive for clinical applications. Our simple two-step one-pot protocol starting from 2-alkylbenzonitriles and Grignard reagents generates stable amino endoperoxides with anticancer properties in good yields. The potential for scalable production and compound stability could suggest an amenable basis for their medicinal realization.

We examined the anticancer effects of amino endoperoxides and their derivatives in the micromolar range, which is higher than some endoperoxide-containing drugs that exert their effects at nanomolar level. However, it should be noted that our treatment duration was significantly shorter $(0.5-24 \mathrm{~h})$ compared with over 2-4 days with other drugs. ${ }^{27,28}$ Therefore, it is conceivable that amino endoperoxides may exert anticancer effects at a much lower concentration matching conventional drug dosage standards if used over a longer period of treatment regime. Furthermore, no apparent toxic symptom was observed in the animals studied, while tumor growth and metastasis were significantly reduced despite the high concentrations used for the treatment. Importantly, it still remains unclear whether other endoperoxide-containing drugs are able to increase anoikis sensitivity of cancer cells. From our findings, we proposed that amino endoperoxides targeting Nox4 are potential antimetastatic drugs. Clearly, future developments of such novel amino endoperoxides to improve bioavailability and efficacy, among others, will be necessary.

The actual mechanism underlying the anticancer role of the prototypical endoperoxide-containing artemisinin and its precise biological target still remains unclear. However, the current consensus of its mechanism of action involves iron(II)mediated release of $\mathrm{ROS}$ and/or carbon centered radicals, ${ }^{15,29,30}$ both of which may have important roles in inducing DNA damage, mitochondrial depolarization, and apoptosis. Recent studies have also implicated the involvement of heme as the mediator of cytotoxicity of artemisinin. ${ }^{16,31}$ Interestingly, we showed that amino endoperoxide (4-Me) induces oxidative apoptosis in tumor cells via a Nox4dependent manner. NADPH oxidases generate ROS in a regulated manner by allowing the transfer of electrons from NADPH via a catalytic core protein Nox containing flavin and heme moieties to molecular oxygen. ${ }^{32}$ In addition, cancer cell lines with high Nox4 expression were sensitive to 4-Me, while a low Nox4 expression was associated with resistance to 4Me. The elevated Nox4 activity in these cancer cell types is required for tumor growth and progression. ${ }^{33,34}$ The amino endoperoxides increased the oxidative stress via a concomitant increase in the production of $\mathrm{H}_{2} \mathrm{O}_{2}, \mathrm{O}_{2}^{-}$and $\cdot \mathrm{OH}$ radicals in cancer cells. It is conceivable that the high $\cdot \mathrm{OH}$ level was due to the reaction between $\mathrm{O}_{2}^{-}$and $\mathrm{H}_{2} \mathrm{O}_{2}$. Notably, although the levels of $\mathrm{H}_{2} \mathrm{O}_{2}$ and $\mathrm{O}_{2}^{-}$were elevated, the $\mathrm{O}_{2}^{-}: \mathrm{H}_{2} \mathrm{O}_{2}$ ratio was reduced, which is likely because Nox4 predominantly produces $\mathrm{H}_{2} \mathrm{O}_{2}$ more than $\mathrm{O}_{2}^{-}$. A high $\mathrm{O}_{2}^{-}: \mathrm{H}_{2} \mathrm{O}_{2}$ ratio is particularly important for cancer cells to sustain their tumorigenicity, anoikis resistance, and thus metastatic 

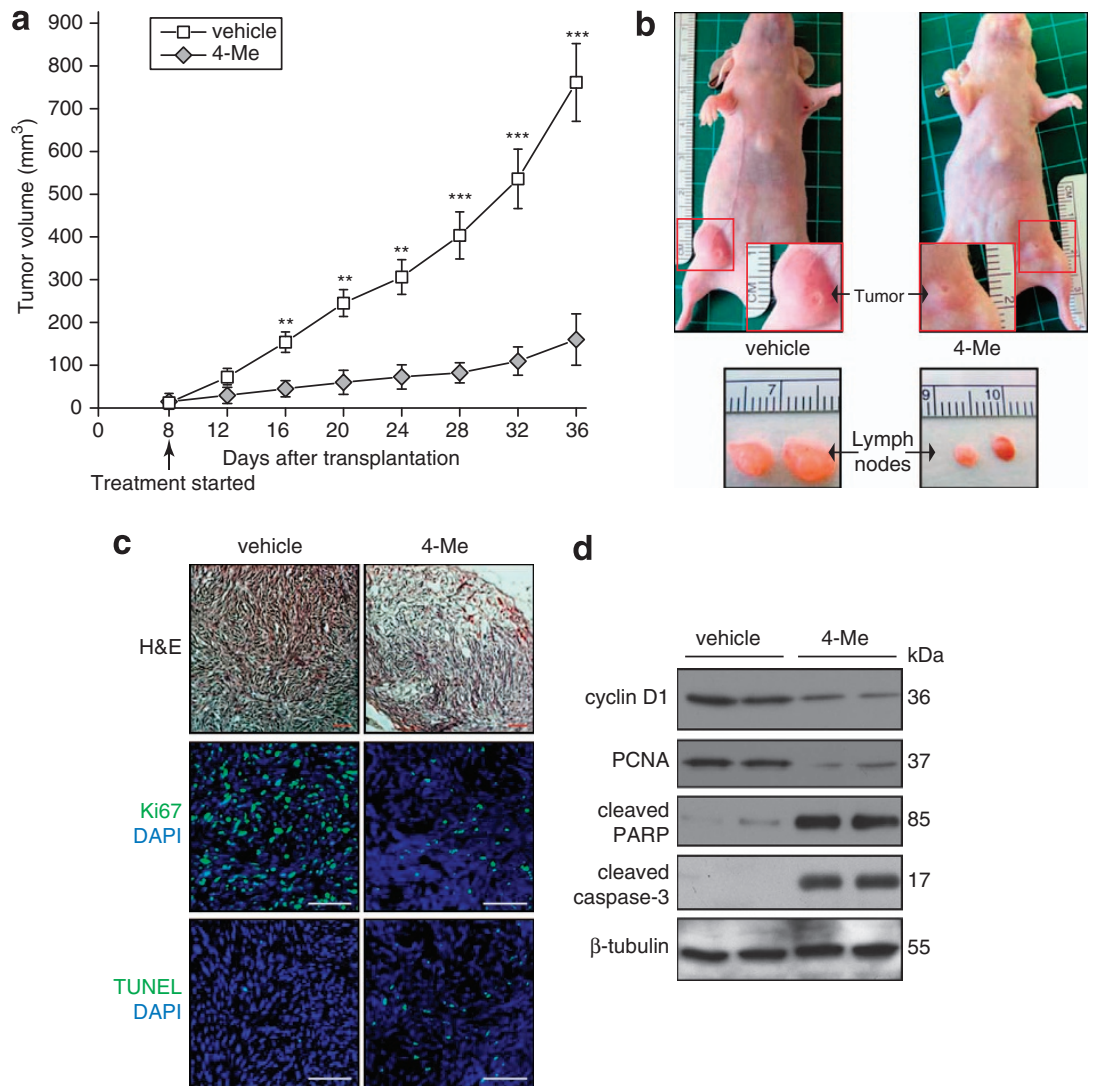

Figure 6 4-Me treatment suppresses MDA-MB-231 orthotopic tumor growth. (a) Orthotopic tumor volumes induced in nude mice by MDA-MB-231 cells. Cells $\left(2 \times 10^{6}\right)$ were inoculated into the fourth mammary fat pad of each mouse. Mice were randomly divided into two groups $(n=5$ per group) at day 8 post cell inoculation. Thereafter, the two groups of mice were treated intraperitoneally with either $3.2 \mathrm{mg} / \mathrm{kg}$ of $4-\mathrm{Me}$ or vehicle every 3 days. Values (means \pm S.D.) are from three measurements of each mouse. ${ }^{* \star} P<0.01$; ${ }^{* \star *} P<0.001$. (b) Representative pictures (top panels) of vehicle control and 4-Me-treated nude mice (day 36) in (a). Higher magnifications of tumor pictures are shown in red rectangles. Bottom panels show enlarged (indicating metastasis) and normal sized lymph nodes in vehicle and 4-Me-treated mice, respectively. (c) Haematoxylin and eosin and immunofluorescence staining of tumor sections from vehicle- and 4-Me-treated nude mice. Proliferating (Ki67) and apoptotic (TUNEL) cells were identified using the indicated antibodies or assay. Sections were counterstained with DAPI (blue). Images were acquired in one z-plane using a LSM710 confocal laser scanning microscope and ZEN 2008 software (Carl Zeiss, Oberkochen, Germany). Scale bars represent $100 \mu \mathrm{m}$. (d) Immunoblots of proliferation (cyclin D1 and PCNA), and apoptosis (cleaved PARP and cleaved caspase-3) markers on tumor biopsies of vehicle and 4-Me-treated nude mice. Immunoblot data are from three independent experiments performed in duplicate. $\beta$-Tubulin serves as a loading and transfer control. (c and $\mathbf{d})$ All experiments were performed using tumor biopsies harvested from mice described in (a) at day 36

potential. ${ }^{6,9,35}$ The role of ROS as a cause or consequence of cell growth or death remains debatable. The findings from clinical trials on the effect of antioxidants on cancer remain inconclusive. $^{36-39}$ Nonetheless, our finding that reducing $\mathrm{O}_{2}^{-}: \mathrm{H}_{2} \mathrm{O}_{2}$ ratio can be manipulated by drugs to elicit cancer cell death suggests that oxidative stress-mediated apoptosis in tumors remains an exciting and viable anticancer strategy and warrants further detailed investigation.

\section{Materials and Methods}

Cell culture. MDA-MB-231 (human breast adenocarcinoma), HEK 293T (human embryonic kidney cells) HaCaT (human keratinocyte), and A-5RT3 (human metastatic squamous cell carcinoma) cells were maintained in Dulbecco's modified Eagle's medium supplemented with 10\% heat-inactivated fetal bovine serum. MCF-10A cells (normal human mammary epithelial cells) were grown in Dulbecco's modified Eagle's medium/F12 supplemented with $5 \%$ horse serum, hydrocortisone $(0.5 \mu \mathrm{g} / \mathrm{ml})$, cholera toxin $(100 \mathrm{ng} / \mathrm{ml})$, insulin $(10 \mu \mathrm{g} / \mathrm{ml})$, and EGF $(20 \mathrm{ng} / \mathrm{ml})$. MKN28 (human gastric carcinoma) cells were maintained in RPMl1640-supplemented $10 \%$ fetal bovine serum. All cells were cultured at $37^{\circ} \mathrm{C}, 5 \% \mathrm{CO}_{2}$, in a $75 \%$ humidified incubator. All the amino endoperoxides and derivatives treatment experiments were performed in cognate serum-free medium.
Apoptosis analysis. To evaluate the cytotoxicity (apoptotic effect) of the novel amino endoperoxides and their derivatives on various cell lines, Annexin V/ propidium iodide $(\mathrm{PI})$ staining coupled with flow cytometry (BD Accuri C6, Accuri Cytometers, Inc., Ann Arbor, MI, USA) was employed. Adhered cells were exposed to each of the amino endoperoxides and derivatives at different concentrations for $30 \mathrm{~min}$, harvested by trypsinization, washed with PBS, and subjected to Annexin V/PI staining for FACS analysis. For anoikis assay, cells were subjected to suspension by seeding cells onto a poly-HEMA-coated plate and treated with endoperoxides as described above before FACS analysis. All experiments were performed in triplicates. Vehicle controls were performed accordingly. Annexin $\mathrm{V}+/ \mathrm{PI}-$ and Annexin $\mathrm{V}+/ \mathrm{PI}+$ cells were considered apoptotic.

ROS measurement. ROS measurement was performed as previously described with modifications. ${ }^{40}$ Briefly, cells were incubated with $10 \mu \mathrm{M}$ CM-H2DCFDA (Invitrogen Life Technologies, Carlsbad, CA, USA) for $30 \mathrm{~min}$ at $37^{\circ} \mathrm{C}$. Next, cells were treated with indicated concentrations of amino endoperoxides and derivatives for $30 \mathrm{~min}$ as described above for FACS analysis. Vehicle controls were performed accordingly. Data were analyzed with Flowjo 7.6. All experiments were performed in triplicates.

Measurement of $\mathrm{O}_{2}^{-}, \mathrm{H}_{2} \mathrm{O}_{2}$, and $\cdot \mathrm{OH}$ levels. Cells were treated with indicated concentrations of amino endoperoxides and their derivatives as described 
above for FACS analysis. Intracellular $\mathrm{O}_{2}^{-}$and $\mathrm{H}_{2} \mathrm{O}_{2}$ levels of the treated cells were measured by MCLA and Amplex Red (Invitrogen Life Technologies), respectively. ${ }^{9}$ The $\mathrm{O}_{2}^{-}: \mathrm{H}_{2} \mathrm{O}_{2}$ ratio was determined by direct comparison with the value of vehicle control cells, which were arbitrarily assigned the value of 1 . We used TA to measure

. OH level. ${ }^{24}$ The hydroxylation of TA yields a stable and highly fluorometric isomer (excitation, $326 \mathrm{~nm}$; emission, $432 \mathrm{~nm}$ ). Hydroxylated TA formed quickly $(<10 \mathrm{~s})$, is resistant to bleaching ( $<5 \%$ change in fluorescence), and permitted detection of $<0.5 \mathrm{pmol} \cdot \mathrm{OH}$. All experiments were performed in triplicates.

EPR measurement of $\mathrm{O}_{2}^{-}$and $\cdot \mathrm{OH}$. EPR measurement of $\cdot \mathrm{OH}$ and $\mathrm{O}_{2}^{-}$were performed as previously described with modifications. ${ }^{9,41}$ Briefly, direct trapping of $\mathrm{O}_{2}^{-}$and $\cdot \mathrm{OH}$ in aqueous media was performed using the spin trap DEPMPO and BMPO, which form relatively stable $\mathrm{O}_{2}^{-}$and $\cdot \mathrm{OH}$ adducts, respectively. EPR spectra were recorded at room temperature with a Bruker D-200ER spectrometer, operating at X-band with a TM 110 cavity with a quartz flat cell. For $\mathrm{O}_{2}^{-}$adduct, the EPR parameters were set at $100 \mathrm{kHz}, \mathrm{X}$-band microwave frequency, $9.5 \mathrm{GHz}$; microwave power, $20 \mathrm{~mW}$; modulation amplitude, $1 \mathrm{G}$; time constant, $160 \mathrm{~s}$; scan time, $50 \mathrm{~s}$; and receiver gain, $5 \times 10^{5}$. EPR signal amplitude at $3480 \mathrm{G}$ represent the pure lines corresponding to the $\mathrm{O}_{2}^{-}$adduct. For $\cdot \mathrm{OH}$ adduct, spectrometer parameters were: modulation frequency, $100 \mathrm{kHz}$; scan range, $100 \mathrm{G}$; field set, $350 \mathrm{G}$; microwave power, $10 \mathrm{~mW}$; modulation amplitude $1 \mathrm{G}$; time constant, $5 \mathrm{~ms}$; and scan time $5.14 \mathrm{~s}$. EPR signal amplitude at $3410 \mathrm{G}$ represent the pure lines corresponding to the. $\mathrm{OH}$ adduct. Tiron and dimethylthiourea were used as negative controls for $\mathrm{O}_{2}^{-}$and $\cdot \mathrm{OH}$ measurements, respectively. The EPR spectra represent the averaged signals of 10 scans. All experiments were performed in triplicates.

Affinity pulldown and LC-MS/MS. Total cell protein lysate was incubated with amino endoperoxide ( $4^{\prime}$-alkyne) for $1 \mathrm{~h}$ to capture its potential intracellular targets, the mixture was next subjected to CLICK-chemistry reaction with biotinconjugated azide as described by the manufacturer (Life Technologies), enabling the affinity pulldown of the molecular complexes of 4-Me and its interacting targets by streptavidin beads. Bound proteins were eluted and subjected to LC-MS/MS as previously described. ${ }^{42}$

Orthotopic tumor model. To investigate the in vivo therapeutic effects of amino endoperoxide (4-Me) on tumor development, an MDA-MB-231 orthotopic nude mice model was used. Two millions of MDA-MB-231 cells were transplanted into the fourth mammary fat pad of each six-week-old female Balb/c athymic nude mouse. The mice were randomly divided into two groups ( $n=5$ each group) 8 days after tumor cells transplantation, and the two groups of mice were treated intraperitoneally with either $3.2 \mathrm{mg} / \mathrm{kg}$ of $4-\mathrm{Me}$ or control vehicle every 3 days. The orthotopic tumors were externally measured with a vernier caliper every other day, and tumor volume was estimated using the equation, $V=\left(L \times W^{2}\right) / 2$, where $L$ and $W$ are the length of the major and minor axis of the tumor, respectively. Mice were killed at the end of the experiment (day 36), and tumors were harvested for further analyses. For toxicology studies, $5 \times(16 \mathrm{mg} / \mathrm{kg})$ and $10 \times(32 \mathrm{mg} / \mathrm{kg})$ more $4-\mathrm{Me}$ were used. The liver, heart, and kidney were harvested at the end of experiment and analyzed for necrosis and cellular apoptosis. All animals were maintained in pathogen-free conditions. The animal studies were approved by the Institutional Animal Care and Use Committee (ARF-SBS/NIE-A0141AZ), Nanyang Technological University, and all experiments were carried out in strict compliance with their regulations. All of the mice were well tolerated without obvious signs of drugrelated toxicity throughout the course of this study.

Statistical analyses. Statistical significance between two groups was analyzed using unpaired nonparametric test (Mann-Whitney test) or with a Student's $t$-test (SPSS, Inc.). All statistical tests were two-sided. A $P$-value of $\leq 0.05$ was considered significant.

\section{Conflict of Interest}

The authors declare no conflict of interest.

Acknowledgements. This work is supported by research grants from Biomedical Research Council (BMRC; 10/1/22/19/644) to NST, and from the Singapore Ministry of Education (Academic Research Fund Tier 2: MOE2010-T2-1 009 to SC, and Academic Research Fund Tier 2: MOE2012-T2-1-014 to SC and
NST). We thank Dr. Yongxin Li and Dr. Rakesh Ganguly (Division of Chemistry and Biological Chemistry, School of Physical and Mathematical Sciences, Nanyang Technological University) for assistance in X-ray crystallographic analysis.

\section{Authors contributions}

$\mathrm{PZ}$ and BMKT carried out the experiments, analyzed the data and PZ wrote the manuscript. BMKT and GYA synthesized and characterized the amino endoperoxides and their derivatives under the supervision of SC, RW, JPC, SF, HCC, and XLW conducted parts of the experiments and collected the data. SC and NST initiated and supervised the project, discussed, and revised the manuscript.

1. Fidler IJ. The pathogenesis of cancer metastasis: the 'seed and soil' hypothesis revisited. Nat Rev Cancer 2003; 3: 453-458.

2. Steeg PS. Tumor metastasis: mechanistic insights and clinical challenges. Nat Med 2006; 12: 895-904.

3. Fidler IJ. Critical determinants of cancer metastasis: rationale for therapy. Cancer Chemother Pharmacol 1999; 43: S3.

4. Hanahan D, Weinberg RA. Hallmarks of cancer: the next generation. Cell 2011; 144: 646674

5. Giannoni E, Buricchi F, Grimaldi G, Parri M, Cialdai F, Taddei ML et al. Redox regulation of anoikis: reactive oxygen species as essential mediators of cell survival. Cell Death Differ 2008; 15: 867-878.

6. Pervaiz S, Clement MV. Superoxide anion: oncogenic reactive oxygen species? Int $J$ Biochem Cell Biol 2007; 39: 1297-1304.

7. Ferraro D, Corso S, Fasano E, Panieri E, Santangelo R, Borrello S et al. Pro-metastatic signaling by c-Met through RAC-1 and reactive oxygen species (ROS). Oncogene 2006; 25: 3689-3698.

8. Suh YA, Arnold RS, Lassegue B, Shi J, Xu X, Sorescu D et al. Cell transformation by the superoxide-generating oxidase Mox1. Nature 1999; 401: 79-82.

9. Zhu P, Tan MJ, Huang RL, Tan CK, Chong HC, Pal M et al. Angiopoietin-like 4 protein elevates the prosurvival intracellular $\mathrm{O} 2(-): \mathrm{H} 2 \mathrm{O} 2$ ratio and confers anoikis resistance to tumors. Cancer Cell 2011; 19: 401-415.

10. Halliwell B, Gutteridge JM. Role of free radicals and catalytic metal ions in human disease: an overview. Methods Enzymol 1990; 186: 1-85.

11. Casteel DA. Peroxy natural products. Nat Prod Rep 1992; 9: 289-312.

12. Tang $Y$, Dong $Y$, Vennerstrom JL. Synthetic peroxides as antimalarials. Med Res Rev 2004; 24: 425-448.

13. O'Neill PM, Posner GH. A medicinal chemistry perspective on artemisinin and related endoperoxides. J Med Chem 2004; 47: 2945-2964.

14. Posner GH, O'Neill PM. Knowledge of the proposed chemical mechanism of action and cytochrome p450 metabolism of antimalarial trioxanes like artemisinin allows rational design of new antimalarial peroxides. Acc Chem Res 2004; 37: 397-404.

15. Mercer AE, Maggs JL, Sun XM, Cohen GM, Chadwick J, O'Neill PM et al. Evidence for the involvement of carbon-centered radicals in the induction of apoptotic cell death by artemisinin compounds. J Biol Chem 2007; 282: 9372-9382.

16. Stockwin LH, Han B, Yu SX, Hollingshead MG, ElSohly MA, Gul W et al. Artemisinin dimer anticancer activity correlates with heme-catalyzed reactive oxygen species generation and endoplasmic reticulum stress induction. Int $J$ Cancer 2009; 125: 1266-1275.

17. Ghorai $\mathrm{P}$, Dussault PH, Hu C. Synthesis of spiro-bisperoxyketals. Org Lett 2008; 10 : 2401-2404.

18. Harris J, Waetzig S, Woerpel K. Palladium (II)-catalyzed cyclization of unsaturated hydroperoxides for the synthesis of 1, 2-dioxanes. Org Lett 2009; 11: 3290-3290.

19. Caja L, Sancho P, Bertran E, Iglesias-Serret D, Gil J, Fabregat I. Overactivation of the MEK/ERK pathway in liver tumor cells confers resistance to TGF-\{beta\}-induced cell death through impairing up-regulation of the NADPH oxidase NOX4. Cancer Res 2009; 69: 7595-7602.

20. Yamaura M, Mitsushita J, Furuta S, Kiniwa Y, Ashida A, Goto Y et al. NADPH oxidase 4 contributes to transformation phenotype of melanoma cells by regulating G2-M cell cycle progression. Cancer Res 2009; 69: 2647-2654.

21. Bedard K, Krause KH. The NOX family of ROS-generating NADPH oxidases: physiology and pathophysiology. Physiol Rev 2007; 87: 245-313.

22. Chance B, Sies H, Boveris A. Hydroperoxide metabolism in mammalian organs. Physiol Rev 1979; 59: 527-605.

23. Wagner BA, Evig CB, Reszka KJ, Buettner GR, Burns CP. Doxorubicin increases intracellular hydrogen peroxide in PC3 prostate cancer cells. Arch Biochem Biophys 2005; 440: $181-190$

24. Yan EB, Unthank JK, Castillo-Melendez M, Miller SL, Langford SJ, Walker DW. Novel method for in vivo hydroxyl radical measurement by microdialysis in fetal sheep brain in utero. J Appl Physiol 2005; 98: 2304-2310.

25. Parkin DM, Bray F, Ferlay J, Pisani P. Global cancer statistics, 2002. CA Cancer J Clin 2005; 55: 74-108.

26. O'Shaughnessy J. Extending survival with chemotherapy in metastatic breast cancer. Oncologist 2005; 10: 20-29. 
27. Takada N, Watanabe M, Yamada A, Suenaga K, Yamada K, Ueda K et al. Isolation and structures of haterumadioxins $A$ and $B$, cytotoxic endoperoxides from the Okinawan sponge Plakortislita. J Nat Prod 2001; 64: 356-359.

28. Davies-Coleman MT, Cantrell CL, Gustafson KR, Beutler JA, Pannell LK, Boyd MR. Stolonic acids $A$ and $B$, new cytotoxic cyclic peroxides from an Indian Ocean ascidian Stolonica species. J Nat Prod 2000; 63: 1411-1413.

29. Efferth T, Benakis A, Romero MR, Tomicic M, Rauh R, Steinbach D et al. Enhancement of cytotoxicity of artemisinins toward cancer cells by ferrous iron. Free Radic Biol Med 2004; 37: $998-1009$

30. Efferth T. Molecular pharmacology and pharmacogenomics of artemisinin and its derivatives in cancer cells. Curr Drug Targets 2006; 7: 407-421.

31. Zhang S, Gerhard GS. Heme mediates cytotoxicity from artemisinin and serves as a general anti-proliferation target. PloS One 2009; 4: e7472.

32. Babior BM. NADPH oxidase: an update. Blood 1999; 93: 1464-1476.

33. Hsieh CH, Shyu WC, Chiang CY, Kuo JW, Shen WC, Liu RS. NADPH oxidase subunit 4 mediated reactive oxygen species contribute to cycling hypoxia-promoted tumor progression in glioblastoma multiforme. PloS One 2011; 6: e23945.

34. Edderkaoui M, Nitsche C, Zheng L, Pandol SJ, Gukovsky I, Gukovskaya AS. NADPH oxidase activation in pancreatic cancer cells is mediated through Akt-dependent upregulation of p22phox. J Biol Chem 2011; 286: 7779-7787.

35. Clement M, Pervaiz S. Intracellular superoxide and hydrogen peroxide concentrations: a critical balance that determines survival or death. Redox Rep 2001; 6: 211-214.

36. Blot WJ, Li JY, Taylor PR, Guo W, Dawsey S, Wang GQ et al. Nutrition intervention trials in Linxian, China: supplementation with specific vitamin/mineral combinations, cancer incidence, and disease-specific mortality in the general population. J Natl Cancer Inst, 1993; 85: 1483-1492.
37. Omenn GS, Goodman G, Thomquist M, Grizzle J, Rosenstock L, Barnhart S et al. The beta-carotene and retinol efficacy trial (CARET) for chemoprevention of lung cancer in high risk populations: smokers and asbestos-exposed workers. Cancer Res 1994; 54: 2038s-2043s.

38. Hennekens $\mathrm{CH}$, Buring JE, Manson JE, Stampfer M, Rosner B, Cook NR et al. Lack of effect of long-term supplementation with beta carotene on the incidence of malignant neoplasms and cardiovascular disease. N Engl J Med 1996; 334: 1145-1149.

39. Lee IM, Cook NR, Manson JE, Buring JE, Hennekens $\mathrm{CH}$. Beta-carotene supplementation and incidence of cancer and cardiovascular disease: the Women's Health Study. J Natl Cancer Inst 1999; 91: 2102-2106.

40. Lam CRI, Tan MJ, Tan SH, Tang MBY, Cheung PCF, Tan NS. TAK1 regulates SCF expression to modulate $\mathrm{PKB} \alpha$ activity that protects keratinocytes from ROS-induced apoptosis. Cell Death Differ 2011; 18: 1120-1129.

41. Spasojevic I. Bioavailability and catalytic properties of copper and iron for Fenton chemistry in human cerebrospinal fluid. Redox Rep 2010; 15: 29-35.

42. Goh YY, Pal M, Chong HC, Zhu P, Tan MJ, Punugu L et al. Angiopoietin-like 4 interacts with matrix proteins to modulate wound healing. J Biol Chem 2010; 285: 32999-33009.

(c) (1) $\Theta$ Cell Death and Disease is an open-access journal published by Nature Publishing Group. This work is licensed under a Creative Commons Attribution-NonCommercialNoDerivs 3.0 Unported License. To view a copy of this license, visit http://creativecommons.org/licenses/by-nc-nd/3.0/

Supplementary Information accompanies this paper on Cell Death and Disease website (http://www.nature.com/cddis) 